\title{
Simulating the Optical Phase Conjugation Phenomenon of Light Multiply Scattered through a Macroscopic Random Medium
}

\author{
Snow H. Tseng ${ }^{*, a, b}$ Meng Cui ${ }^{\mathrm{b}}$, Changhuei Yang ${ }^{\mathrm{b}}$ \\ ${ }^{\mathrm{a}}$ Graduate Institute of Photonics and Optoelectronics, and \\ Department of Electrical Engineering, \\ National Taiwan University, Taipei, Taiwan 106 \\ ${ }^{\mathrm{b}}$ Department of Electrical Engineering and Department of Bioengineering, \\ California Institute of Technology, Pasadena, CA. 91125
}

\begin{abstract}
The light scattering effects of turbid media causing opacity may be undone via Optical Phase Conjugation (OPC). Here we rigorously simulate light scattering through a macroscopic random using the pseudospectral time-domain (PSTD) technique. The OPC phenomenon of multiply scattered light can be quantitatively analyzed which is not feasible otherwise. Specifically, factors affecting the electromagnetic energy propagation and refocusing phenomenon is analyzed. The reported simulation study allows accurate characterization of the optical properties of the OPC phenomenon for practical biomedical applications.
\end{abstract}

Keywords: optical phase conjugation, PSTD, random media, multiple scattering

\section{INTRODUCTION}

Realization of the turbidity suppression optical phase conjugation (TS-OPC) has been reported [1]. In addition to the optical phase conjugation (OPC) refocusing of light through random media and recovering the original incident light, the experiment revealed several interesting phenomena that is not well understood. One of the interesting phenomena is that the OPC refocused beam width is approximately the same for various thicknesses of the random medium.

As we have previously shown [2], without any scattering medium, the OPC refocusing effect of light is perfect. For light that scattered through a random medium, the OPC refocusing effect is not perfect; the refocused light pulse becomes blurry, however, the pulse width remains roughly unchanged regardless of the random medium. It is unclear why the OPC refocused pulse width doesn't change significantly. It is understandable that for optically thicker random medium, the refocused intensity decreases, but is the degradation of the OPC refocused light a direct consequence of $\mu_{s}$ ? Our goal is to address this question by simulations.

\section{METHODS}

The Pseudospectral time-domain (PSTD) technique is capable of rigorously simulating light scattering problems of macroscopic dimensions based upon the fundamental electromagnetic principles-Maxwell's equations. We employ the PSTD technique [3] to simulate an optical experiment of a 2-D phase conjugate mirror (PCM) as shown in Fig. 1. The simulation is performed with a spatial resolution of $0.33 \mu \mathrm{m}$ and temporal resolution of $\Delta t=0.05 \mathrm{fs}$. A scattering medium consisting of randomly positioned dielectric cylinders is placed adjacent to a PCM in vacuum. The random medium is illuminated by an incident light pulse; the light pulse scatters through the random medium via complex optical paths. For light impinging the PCM, the $\mathbf{E}$-field and D-field components are manually inverted:

$$
\mathbf{E} \rightarrow-\mathbf{E}, \quad \mathbf{D} \rightarrow-\mathbf{D}
$$

Biomedical Applications of Light Scattering IV, edited by Adam P. Wax, Vadim Backman,

Proc. of SPIE Vol. 7573, 757302 - @ 2010 SPIE · CCC code: 1605-7422/10/\$18 · doi: 10.1117/12.839994

Proc. of SPIE Vol. $7573757302-1$ 
with the magnetic field $\mathbf{H}$ and magnetic induction $\mathbf{B}$ remain unchanged. As a result, the inverted light will continue to propagate in reverse direction, simulating the OPC refocusing effect of a phase conjugate mirror. Initial simulations show results consistent with experimental results; a detailed description of the PSTD simulation of OPC can be found in [2].

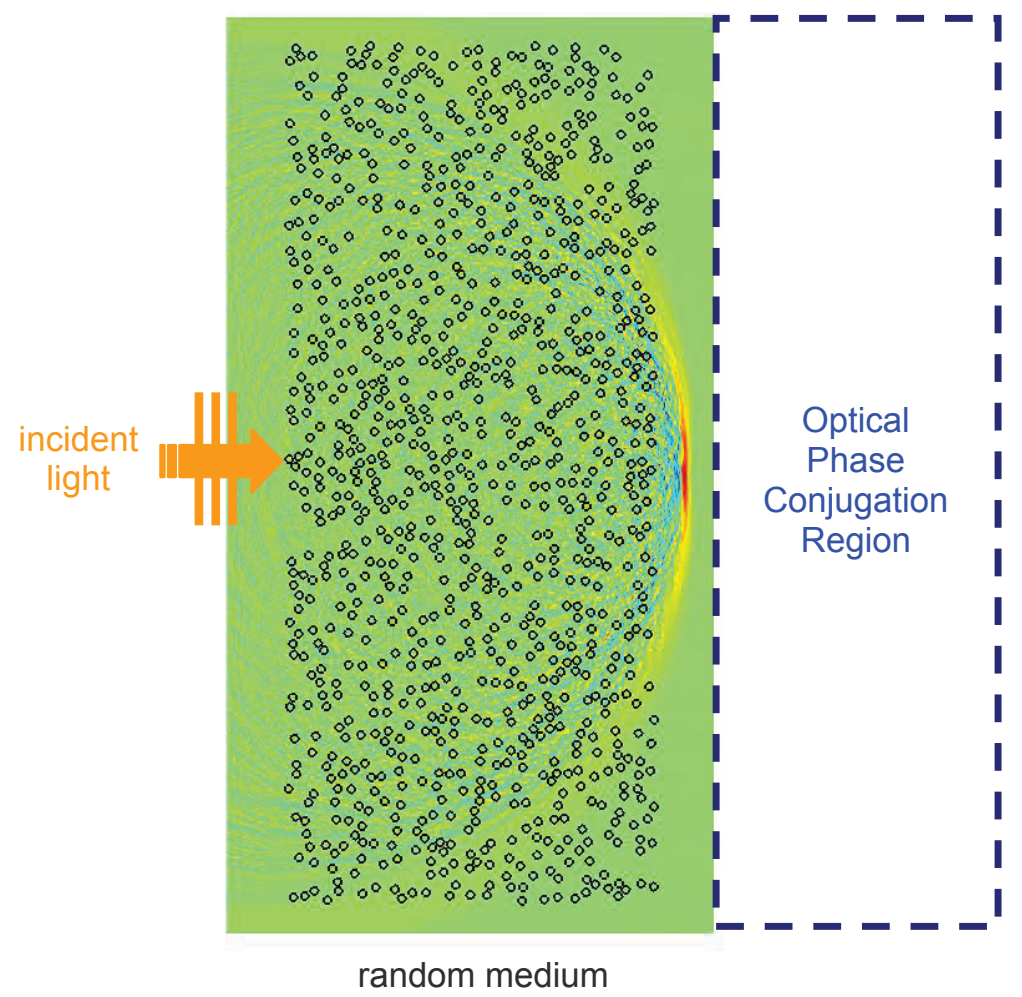

Fig. 1. Simulating the optical characteristics of a phase conjugate mirror (PCM). A light pulse scatters through a random medium consisting of $N$ randomly-positioned, dielectric cylinders, and impinges on a PCM. The OPC effect of a PCM is simulated by inverting the E field component, causing the light pulse to reverse propagation, back-trace its trajectory, and refocus at its origin.

\section{RESULTS}

We investigate the effect of optical thickness of the random medium is increased with an increase of number of cylinders within the random medium. As shown in Fig. 2, the OPC refocused pulse amplitude decreases with increasing thickness of the random medium. However, notice that the cross-sectional width of the OPC refocused light pulse remains approximately unchanged. Our simulation findings agree with experimental findings [1].

Next, instead of varying the physical thickness, we fix the thickness and vary the number density of cylinders by packing more cylinders within the random medium. With an increased number of cylinders within the cluster, the scattering coefficient $\left(\mu_{s}\right)$ of the random medium is increased. It is found that the OPC refocusing phenomenon is related to the optical thickness defined as:

$$
\text { Optical thickness } \equiv \text { (geometrical thickness) / (scattering mean free path) }
$$

As the optical thickness increases with physical thickness or number density of the cylinders, the OPC refocused pulse deteriorates. However, it appears that the width of the OPC refocused light is not directly affected by the specific 
random medium. For different thickness or different number density of the random medium, the OPC width of the OPC refocused light pulse remained roughly the same, as shown both by experiment and simulation [1] [2].

(a)

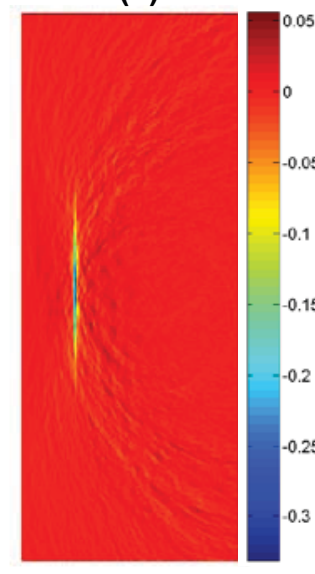

(b)

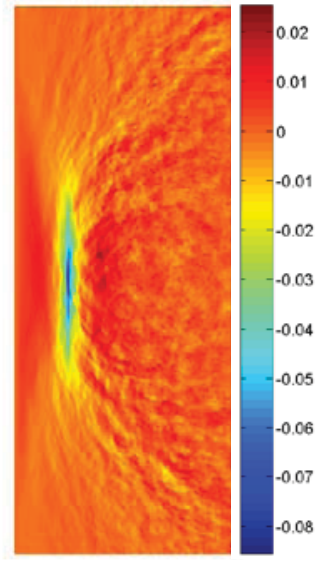

(c)

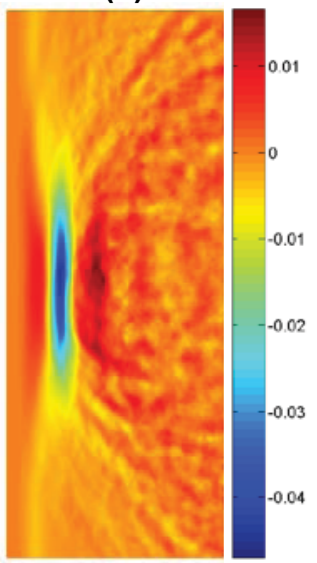

(d)

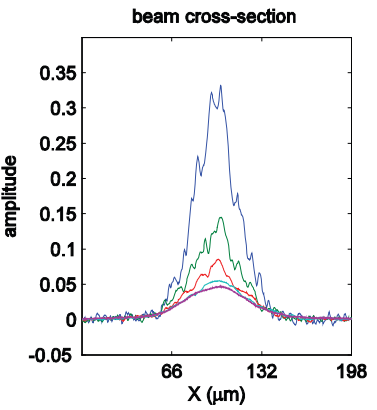

Fig. 2. The OPC refocusing effect deteriorates with increasing scattering coefficient of the random medium. The scattering coefficient $\left(\mu_{s}\right)$ of the random medium is varied by changing the number of dielectric cylinders. Amplitude of the OPC refocused pulse amplitude is plotted in (d) (from bottom to top) for various $\mu_{s}$ (for $\lambda=1 \mu \mathrm{m}$ ) $=0.1291,0.1033,0.0775,0.0516$, and $0.0258, \mu \mathrm{m}^{-1}$. The pulse width remains roughly unchanged.

The degradation of the OPC refocusing phenomenon may be a result of incomplete phase conjugation of the optical field [2]. In theory, the presence of a random medium only distorts the optical path but shouldn't cause loss. We suspect the degradation of OPC refocused light may be accounted for by the fraction of light that is scattered away from the PCM. If all scattered light impinges a PCM, the OPC refocusing phenomenon may be perfect with no degradation.

In order to determine the cause of degradation of the OPC refocusing effect, we propose to have PCM mirror in every light scattering angle fully surrounding the random medium, so that all scattered light will undergo OPC and backtrace its previous optical path. In experiment, it is difficult to carry out having PCM mirror in every scattering direction, whereas in simulations, this can be easily achieved by inverting the phase of the $\mathbf{E}$-field and $\mathbf{D}$-field of the entire space. The full-space OPC is equivalent to having PCM in every light scattering direction where all scattered light undergoes OPC. As shown in Fig. 3, the full-space OPC results in perfectly refocused light pulses identical to the original incident pulses (with a 180-degree phase difference). The simulation results confirm that if all of the scattered light undergoes $\mathrm{OPC}$, the OPC refocusing effect will be identical to the incident pulse.

\section{SUMMARY}

As a consequence, the width of the OPC refocused light pulse is determined by the incident pulse. If the OPC refocusing effect is perfect, the OPC refocused light pulse will have a width identical to the incident pulse. However, degradation of the OPC phenomenon occurs due to the fact that only a fraction of light impinges the PCM and is refocused. A different optical thickness of the random medium will cause different degrees of light scattered away from the PCM and lost. Therefore, the width of the OPC refocused light pulse is approximately the same as it is dictated by the incident light pulse, with deterioration due to incomplete phase conjugate light field. 
(a)

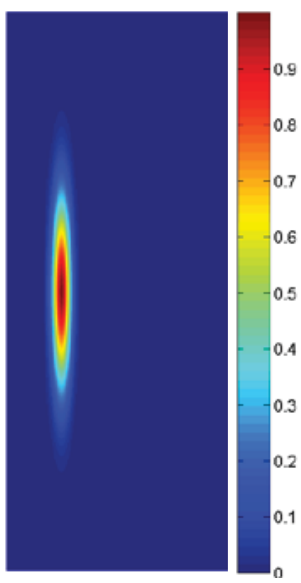

(b)

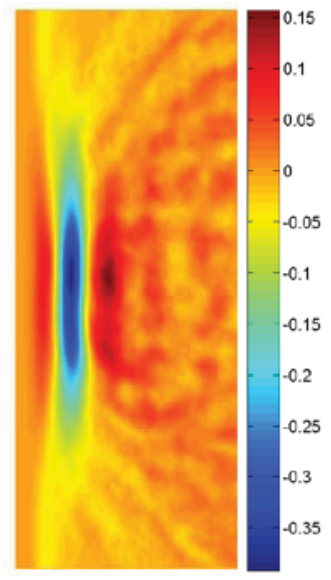

(c)

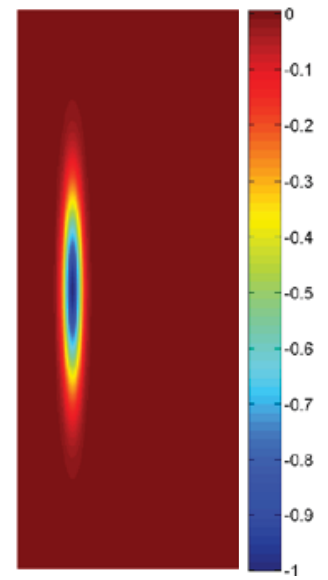

Fig. 3. Full-space OPC. Having phase conjugate mirror in every light scattering direction can be simulated by inverting the poynting vector of the scattered light in the entire space. The OPC refocused beams from a phase conjugate mirror are shown in (a)-(c). Notice that the full-space OPC reconstructs the light pulse to its initial amplitude, showing that all energy has been refocused.

In this manuscript, we simulate the OPC refocused phenomenon where the light intensity decreases with increasing thickness of the scattering medium, whereas the OPC refocused cross-sectional width remain unchanged. Simulation results show that the OPC refocused intensity decrease is a consequence of scattering of the turbid medium. Furthermore, we show that the cross-sectional width of the OPC refocused light pulse is insensitive to the optical thickness of the scattering medium. By simulating OPC effect for the entire electromagnetic field in space, we have shown that the presence of the random medium only distorts the optical paths but does not cause degradation of the OPC phenomenon. We have shown that the degradation of the OPC refocusing phenomenon is caused by incomplete OPC of the optical field: as the thickness or number density of cylinders of the random medium increases, more scattering occurs causing more light scattered away into other directions. If all the scattered light undergoes phase inversion, the OPC refocused light pulse will be lossless and identical to the incident light pulse (with a 180-degree phase difference), which further explains why the width of the OPC refocused light pulse is approximately the same regardless of the specific random medium. Based upon Maxwell's equations, the OPC phenomenon for arbitrary geometry can be accurately analyzed by means of the reported PSTD simulation.

\section{ACKNOWLEDGEMENTS}

The authors thank the Taiwan National Science Council Grant NSC 96-2112-M-002-028-MY3 for the support of this research. In addition, the authors thank the National Taiwan University Computing Center for providing the computing facilities.

\section{REFERENCES}

1. Z. Yaqoob, D. Psaltis, M.S. Feld, and C. Yang, "Optical phase conjugation for turbidity suppression in biological samples," Nature Photonics, 2008. 2: p. 110-115.

2. S.H. Tseng and C. Yang, "2-D PSTD Simulation of optical phase conjugation for turbidity suppression," Optics Express, 2007. 15(24): p. 16005-16016.

3. Q.H. Liu, "Large-scale simulations of electromagnetic and acoustic measurements using the pseudospectral time-domain (PSTD) algorithm," IEEE Transactions on Geoscience and Remote Sensing, 1999. 37(2): p. 917-926. 